\title{
ESTRATEGIA CORPORATIVA Y COMUNICACIÓN DE LA RESPONSABILIDAD SOCIAL EMPRESARIAL
}

\author{
Corporate strategy and communication of corporate social responsibility
}

Estratégia corporativa e comunicação da responsabilidade social empresarial

María Concepción Parra-Meroño

Universidad Catolica San Antonio de Murcia

mcparra@ucam.edu

José Gabriel Mira-Agulló

Universidad Catolica San Antonio de Murcia

jgmira@ucam.edu

\section{Resumen}

La Cultura y Filosofía empresarial ha evolucionado significativamente en las últimas décadas. La gestión empresarial ha dejado de estar centrada en aspectos netamente económicos, para evolucionar a componentes sociales y políticos relevantes, atribuyéndole también una responsabilidad ética con la sociedad, el entorno y el medio ambiente. Esta labor de responsabilidad frente a la sociedad es un aspecto fundamental que actualmente se está gestionando y se ha integrado en la estructura corporativa de la empresa como un factor estratégico de crecimiento y desarrollo. Este trabajo, basándose en la situación de la Responsabilidad Social Corporativa en España en la actualidad, pone en valor la importancia que ha de dar la comunicación corporativa a la comunicación de la responsabilidad social empresarial.

Palabras clave: Comunicación estratégica. Comunicación corporativa. Gestión RSC.

\begin{abstract}
Culture and Business philosophy has evolved significantly in recent decades. Business management has ceased to be focused on purely economic aspects to evolve into relevant social and political components and undertaking an ethical responsibility towards society and the environment. This responsibility towards society is a fundamental aspect that is currently being managed and has been integrated into the corporate structure of the company as a strategic factor of growth and development. Based on the current situation of Corporate Social Responsibility in Spain, this paper emphasizes the importance that corporate communication should place on the communication of corporate social responsibility.
\end{abstract}

Key words: Strategy communication. Corporate communication. CSR management.

\section{Resumo}

A Cultura e a Filosofia empresarial têm evoluído significativamente nas últimas décadas. A gestão empresarial tem deixado de estar centrada nos aspectos meramente económicos, para 
evoluir à componentes sociais e políticos relevantes, atribuindo-se também a uma responsabilidade ética com a sociedade, o entorno e o meio ambiente. Esta tarefa de responsabilidade frente à sociedade é um aspecto fundamental que atualmente se está gerindo e se tem integrado na estrutura corporativa da empresa como um fator estratégico de crescimento e desenvolvimento. Este trabalho, baseando-se na situação de Responsabilidade Social Corporativa na Espanha atual, põe em questão a importância que há de dar a comunicação corporativa à comunicação de responsabilidade social empresarial.

Palavras-chave: Comunicação estratégica. Comunicação Corporativa. Gestão RSC.

\section{INTRODUCCIÓN}

La Comunicación Corporativa es todo lo que la empresa dice sobre sí misma. Con este tipo de comunicación se ejecutan acciones, se distribuyen las responsabilidades, se representa y transmite su realidad (BRICEÑO, MEJÍAS y MORENO, 2010). Integra todas las formas y medios que utiliza la empresa para reforzar y potenciar su identidad, y contribuye a la consolidación y mejora de su imagen corporativa (lo que el público identifica con ella) y su reputación. Es decir, convierte la identidad corporativa en imagen corporativa y cuando éstas se mantienen alineadas en el tiempo se refuerza su reputación.

Garrido (2004) define la estrategia de comunicación como un marco integrador, que unifica la acción comunicativa de la empresa, de acuerdo al diseño de objetivos establecidos a largo plazo. Mazo (2001) añade que los objetivos estratégicos no solo deben ser fijados por la Alta Dirección, sino que deben participar en su establecimiento todos los grupos de interés que se puedan ver afectados en el proceso, positiva o negativamente, por sus decisiones o actuaciones. Idea que refuerza Pizzolante (2006) al exponer que, en esta comunicación estratégica, el equipo humano debe estar alineado hacía el objetivo común y en una misma dirección. La identidad y su realidad deben ser expresadas con auténticos comportamientos de sus directivos y empleados, si no es así los grupos de interés perderán la confianza y no se conseguirá la diferenciación competitiva que se persiga, como elemento de diferenciación.

La empresa privada ha dejado de desempeñar un papel económico individual y ha adquirido una representatividad social activa en su relación con el resto de la sociedad y el Estado, adquiriendo cada vez más fuerza componentes de carácter social y político. Así, a través de la implantación de la Responsabilidad Social Corporativa (RSC), se produce el cambio del modelo de gestión con el que se busca generar un nuevo valor agregado, mejorando la reputación de la empresa, y provocando con ello nuevos cauces de obtención de 
utilidades que permitan su subsistencia en el tiempo, dejando un poco de lado los aspectos de rentabilidad económica y calidad de productos como factores esenciales, en los que basar la obtención de beneficios.

Así, las empresas incorporan, asumen y gestionan las externalidades que provocan con su actividad, tanto a nivel económico como social o medioambiental, mediante el contacto directo con sus grupos de interés. Para ello se fortalecen los canales de diálogo estableciendo compromisos con ellos y resolviendo las expectativas que se depositan en ellas. Para conseguir estas premisas, la comunicación de la RSC ha pasado a formar parte de la estrategia comunicativa corporativa, integrándose en la filosofía y la cultura empresarial al mismo nivel que otros aspectos económicos, antes predominantes y fundamentales.

El análisis de la comunicación de la RSC, como factor clave en el desarrollo de la estrategia corporativa empresarial, se acomete en este trabajo presentando el papel de la empresa privada como nuevo actor social que adquiere nuevas responsabilidades; justificando su adopción como enfoque de la gestión empresarial; debatiendo sobre la importancia de comunicar la responsabilidad social y relacionando la comunicación corporativa con la responsabilidad social empresarial. Finalmente se expone la situación de la RSC en España a partir del estudio sobre la RSC realizado por el Club de Excelencia en Sostenibilidad en 2016.

\section{DESARROLLO}

\subsection{La RSC como nuevo enfoque de la gestión empresarial}

La actual coyuntura económica y la crisis que viene padeciendo y arrastrando la economía en los últimos años, ha influido positivamente en la potenciación de la RSC, obligando a las empresas a buscar caminos alternativos que potencien su imagen, ante la sociedad, con el fin de asegurar su supervivencia. Así mismo, la liberalización, junto con la globalización de la economía ha provocado que corporaciones multinacionales hayan ido adquiriendo un papel activo en las actuaciones públicas, influyendo en las políticas de los Estados, con lo que la responsabilidad empresarial se ha ampliado y con ello sus escenarios de actuación (CARRILLO, 2015). Así, se acepta la idea de que las organizaciones empresariales han pasado a ser un actor influyente en la interacción social con el medio, ya que los impactos que provocan en el entorno, natural y social, ya son visibles y medibles y la obtención de un óptimo social no podría alcanzarse si las externalidades que provocan, en su devenir, no son asumidas e interiorizadas. 
La asunción por parte de las empresas de aceptar y gestionar las externalidades que provocan, tanto a nivel social como económico o medioambiental, en un costo significativo. Sin embargo, empiezan a percatarse de que las acciones que ponen en marcha con este fin son también fuente de incremento de beneficios. El aumento de la reputación y la imagen corporativa que provocan, estimulan sinergias positivas que hacen que el resultado final, coste-beneficio, sea más que satisfactorio en conjunto, ya que favorece su relación con el público, conlleva el mantenimiento de clientes y también facilita el acceso a nuevos mercados (ANCOS, 2010). Así, Kotler, Hessekiel y Lee (2012) van un paso más allá y añaden a la búsqueda de la excelencia (hacer lo mejor), el concepto de responsabilidad (hacer lo bueno). De este modo la creación de valor se puede lograr con políticas de RSC dirigidas a todos los stakeholders (Figura 1).

Figura 1. Creación de Valor

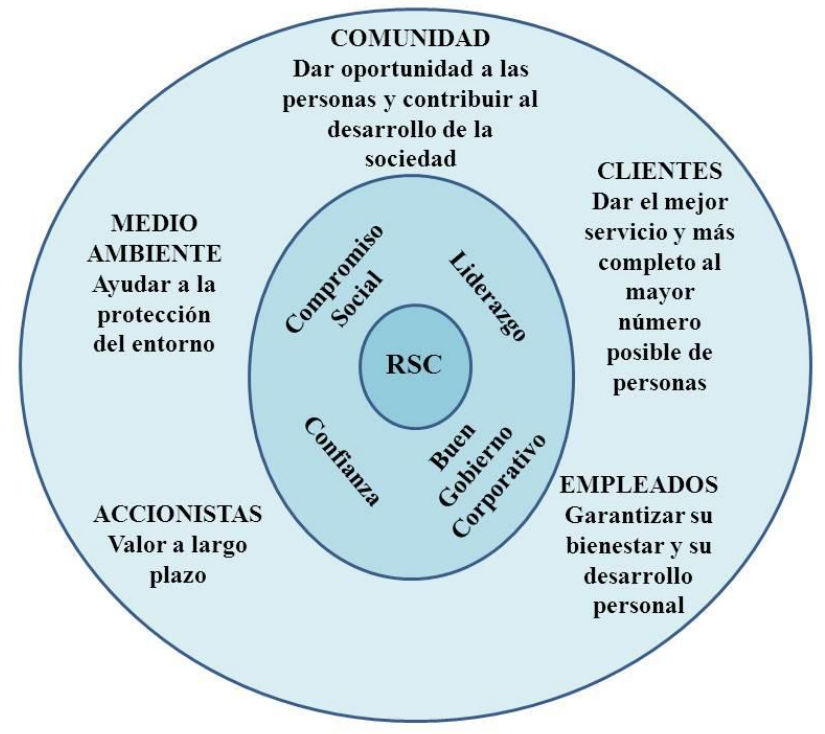

Fuente: Kotler, P., Hessekiel, D. y Lee, N. (2012). Lo bueno funciona. Madrid: LID Editorial, pp.282

A la hora de sopesar el análisis coste-beneficio de la divulgación voluntaria de la RSC apuntan Vicente, Balderas, Ruiz \& Tamayo (2007), como factor principal de justificación del aumento de competitividad, al interés por cuestiones de imagen corporativa y la reputación empresarial. Y señalan diversos factores a favor como: la mejora de la imagen corporativa (con la consiguiente atracción de empleados, clientes e inversores adecuados), adición de valor intangible a los productos de la empresa, otorgar legitimidad ante grupos de interés, conocer las demandas de los clientes actuales y aumentar el orgullo de pertenencia. En 
referencia a los costes señalan: la asunción de costes no obligatorios, la dificultad de la gestión administrativa y el posible escaso interés de los participantes.

Así, una vez concienciados de los beneficios que van implícitos a asumir políticas de RSC, y de integrar adecuadamente estás en la estrategia empresarial y a nivel operativo, aparecen ventajas competitivas que posibilitan un mejor posicionamiento estratégico (ANCOS, 2010). Se debe diseñar un proceso de gestión en el que todas las actividades sean acordes con la RSC, integrando primeramente en la gestión interna preocupaciones sociales, laborales y medioambientales, incluyendo y teniendo en cuenta a los interlocutores implicadas en ellas, para posteriormente incluir a los externos, con el fin de presentarse como una entidad responsable y comprometida en el medio en el que está inmersa. Es decir, conseguir primero que la RSC se integre en la empresa a nivel interno, para a posteriori comunicar a los grupos de interés las acciones y caminos tomados y alcanzar el fin perseguido.

Ancos (2010) distingue en el proceso de implantación de la RSC en la empresa cuatro fases diferenciadas. La primera sería una fase de autodiagnóstico inicial. La segunda comprendería la recopilación de la información de las percepciones que tienen los grupos de interés (stakeholders) en relación con las acciones y actuaciones de la empresa (siempre con relación a la llamada Triple Bottom Line o triple línea básica) en referencia a objetivos económicos, sociales y medioambientales y favoreciendo el espacio de discusión necesario. La tercera fase comprendería el análisis y la elaboración del plan de actuación a llevar a cabo, mediante el establecimiento de indicadores y baremos que encaucen la realización de las actividades o acciones. Y por último, la fase de implantación y control, que conllevaría la aplicación de las acciones y el análisis de la consecución de resultados y objetivos que permitan una retroalimentación que conlleve un proceso de mejora continua. En el proceso de diseño del plan de actuación y posterior desarrollo y control, deben tener cabida todos los interlocutores o grupos de interés involucrados en la actividad de la empresa (equipos multidisciplinares y multidepartamentales en conjunto).

El interés en el diseño de este Sistema de Gestión de Responsabilidad Social (SGRS) debe ser crear una gran marca de identidad corporativa que pueda establecer algunas distinciones con otras empresas y que proporcione la ventaja competitiva correspondiente (VÁZQUEZ y POLO, 2013). 


\subsection{La comunicación de la RSC}

La comunicación de la RSC consiste en incorporarla en los canales y mecanismos de comunicación existentes (VÁZQUEZ y POLO, 2013), de forma que su inclusión no suponga un esfuerzo demasiado grande en organizaciones pequeñas y medianas. Los canales de comunicación a utilizar pueden ser variados, tal y como apuntan diversos autores, como por ejemplo a través de sus propias páginas web, insertando sus informaciones en una web global o utilizando medios más informales. En cuanto al tipo de formato a elegir, también podría ser variado, mencionando a modo de ejemplo y sin pretender una relación exhaustiva: informes sociales, informes temáticos, declaraciones de intenciones y códigos de conducta, eventos, premios, etc. Todo ello, en honor a la transparencia y la rendición de cuentas voluntaria en el acometimiento de estas acciones y ante la creciente atención que en la actualidad se presta a este aspecto (ARVIDDSON, 2010).

La integración de la comunicación de la RSC implica un cambio en la gestión estratégica organizacional estableciendo un SGRS, tal y como apuntan Vázquez y Polo (2013), y entendiendo éste como el desarrollo del esquema de la comunicación sostenible, es decir la forma en cómo la empresa dirige y supervisa las diferentes actividades en materia de RSC, recogiendo los principales indicadores de desempeño económico, social y medioambiental, así como el seguimiento periódico de su evolución.

El establecimiento de un SGRS es determinante (VICENTE et al., 2007), ya que muestra la trascendencia de la comunicación de las actividades para transmitir credibilidad; a través de la transparencia, la cantidad, la calidad y la fiabilidad de la información vertida. De este modo, señala Mouriz (2007) que es necesaria la formalización de políticas y sistemas de gestión en el ámbito económico, social y medioambiental, como medio de transmisión de transparencia informativa en la revelación de resultados alcanzados, que permitirán la adecuada toma de decisiones en materia de RSC y su comunicación (VIDAL, BULL \& KOZAK, 2010). Y todo ello sin perder de vista la voluntariedad de la divulgación de información que supone la comunicación de la RSC (Figura 2). 
Figura 2. Modelo de comunicación propuesto por Vázquez y Polo (2013)

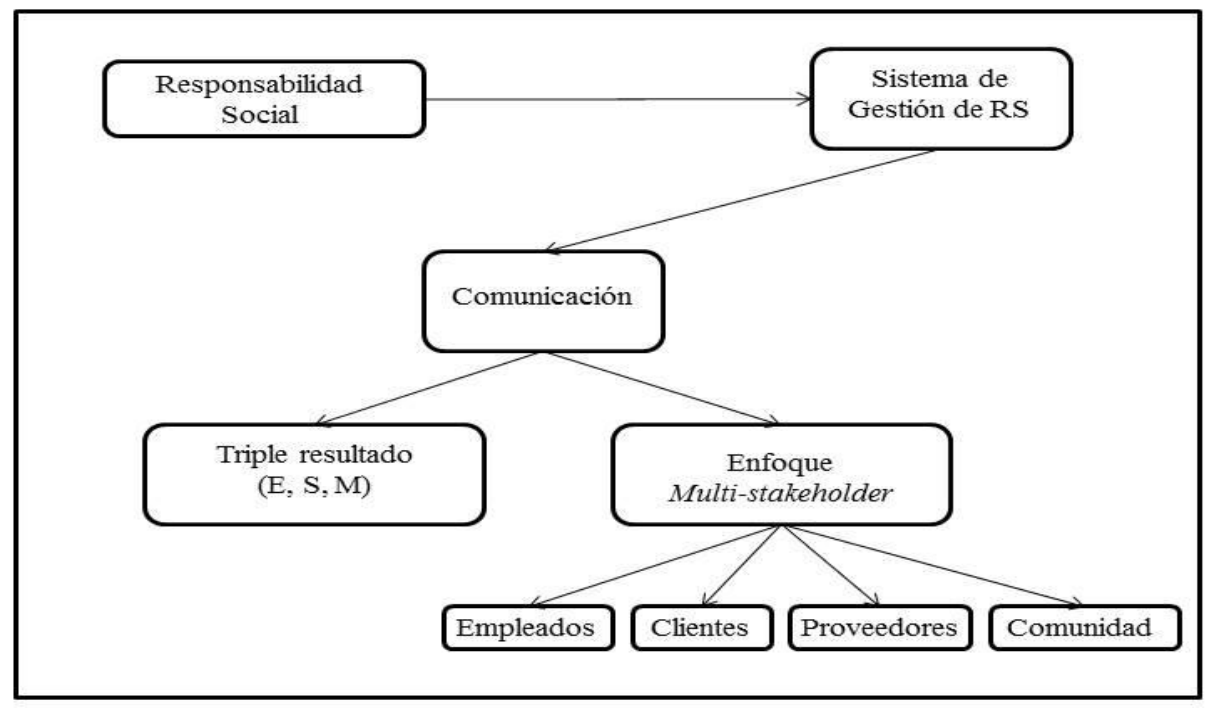

Fuente: Vázquez, D. G., y Polo, F. C. (2013). Diseño de un sistema de comunicación eficaz para la responsabilidad social en pymes. Información Comercial Española, ICE: Revista de economía, 872

El interés en el diseño de este modelo (VÁZQUEZ y POLO, 2013), es crear una gran marca de identidad corporativa que pueda establecer algunas distinciones con otras empresas y que proporcione la ventaja competitiva correspondiente. La comunicación eficaz de la RSC es clave en el proceso de asunción en la empresa, por lo tanto, debe ir dirigida a los principales colectivos con los que esta se relaciona, lo que motivará que se empleen distintos medios de comunicación dependiendo del grupo de interés al que se dirija. La Unión Europea (UE) (2005) define la comunicación eficaz como aquella que garantiza que sus destinatarios reciben realmente el mensaje y, por ello, requiere de todos los esfuerzos necesarios para su fin.

La potenciación de la comunicación de la RSC se debe asemejar a la de un área estratégica, en la misma línea que el Marketing, los Recursos Humanos, las Finanzas o cualquier función tradicional dependiente del gobierno de la empresa (ARGENTI, 2013). De esta forma, se pueden eliminar algunas de las trabas que demuestra el bajo tratamiento de la comunicación por parte de las pymes (por la limitación de recursos disponibles: técnicos, económicos o de personal) y así, asimilar esta herramienta más como una filosofía empresarial, que como un gasto. En definitiva, no solamente hay que realizar acciones y estar comprometido con la RSC, sino que además hay que revelar la actuación responsable de la empresa. La RSC no es solamente un fin en sí, una filosofía y cultura empresarial, también es 
un medio de reconocimiento que, debidamente comunicado, puede reforzar la posición de la empresa a largo plazo con sus grupos de interés y por lo tanto afianzar su supervivencia y la obtención de mejores resultados económicos. Vázquez y Polo (2013) proponen un modelo de comunicación eficaz que se compone de las siguientes fases: (1) Diagnóstico previo: donde la empresa analiza su situación de partida y detecta las necesidades y puntos de mejora. (2) Plan de acción: donde la empresa detectará a los grupos estratégicos a los que se va a dirigir y establecerá los objetivos estratégicos a seguir. (3) Comunicación: donde la empresa se cuestionará ¿Qué? ¿Por qué?, ¿A quién? y ¿Cómo comunicar? (4) Evaluación y análisis de resultados: donde analizará los resultados obtenidos que le permitirán realizar los ajustes necesarios para establecer un proceso de mejora continua, con el fin de obtener de la manera más eficaz y eficiente el objetivo perseguido.

La comunicación es parte de la RSC (BRICEÑO et al., 2010), con ella se transmite la realidad de empresarial a las diferentes audiencias que están en contacto con ella (interna, externa y pública en general). Con la RSC la organización expresa su filosofía, su cultura, su identidad e imagen corporativa, por lo que pasa a ser una variable fundamental en el desarrollo de su activo (intangible) y refuerza su reputación y la confianza en la consecución de sus objetivos.

El libro Verde de la UE (2001) define la RSC como "la integración voluntaria por parte de las empresas, de las preocupaciones sociales y medioambientales a sus operaciones comerciales y sus relaciones con sus interlocutores" (p.7). De ella se extraen una serie de aspectos relevantes como son: la voluntariedad de su integración en la estrategia corporativa, la asunción de las preocupaciones sociales y medioambientales, más allá de los requerimientos legales y administrativos, $\mathrm{y}$, sobre todo, el establecimiento de relaciones con los distintos interlocutores que se relacionan con la organización para que participen en la elaboración y aplicación de los principios que se establezcan.

Las empresas que son socialmente responsables gozan de una reputación más favorable que se muestra en la mayor lealtad de sus clientes, en la potenciación del orgullo de pertenencia de sus trabajadores, en la confianza que reciben de los mercados financieros donde actúan y en sus relaciones con las instituciones públicas (BRICEÑO et al., 2010). Este aumento de la confianza es un factor que es percibido por los empresarios y la alta dirección, con lo que aumenta la consciencia de la importancia que tiene el desarrollo de este ámbito y la notoriedad que la comunicación correcta conlleva en la proyección futura de la organización. 
Para llevar a cabo esta labor social responsable, la empresa debe asignar los recursos necesarios para atacar problemas sociales particulares en distintas áreas, supliendo o reforzando en algunos casos al Estado. Y por ello, Pizzolante (2006) afirma que se convierte en una herramienta de Comunicación Organizacional, bajo distintos puntos de vista:

1. Administrativa, puesto que optimiza y precisa los flujos de información, delimita la toma de decisiones estratégicas y potencia la conformación de grupo y la resolución de conflictos.

2. Competitiva, porque aumenta la confianza de los stakeholders al incrementar la transparencia de su administración mostrando claramente comportamientos y actitudes.

3. De imagen de marca y posicionamiento, al añadir un valor agregado mayor al mero suministro de buenos productos o servicios.

4. Como herramienta intensivadora y activadora de la actividad empresarial, potenciando aspectos de responsabilidad junto a la mejora e incremento de bienestar de los grupos de interés y la comunidad.

\subsection{Situación de la RSC en España}

Actualmente la Responsabilidad Social Corporativa está ligada a la voluntariedad de las empresas en muchos países. Si bien, la actividad normativa en España y en Europa está contribuyendo a la implantación y la asunción de acciones de RSC cada vez en mayor medida por parte de las empresas.

España desde la creación del Consejo Estatal de Responsabilidad Social de las Empresas (CERSE), según Real Decreto 221/2008, de 15 de febrero, se convierte en un país pionero en la aplicación de la RSC en el contexto europeo, hecho que se ha sido reforzado con la aprobación de la Ley 2/2011 de Economía Sostenible, de 4 de marzo.

Este Consejo nace como un órgano asesor y consultivo del Gobierno, adscrito al Ministerio de Empleo y Seguridad Social, para establecer el marco de referencia de éste en materia de Responsabilidad Social, impulsando y fomentando las políticas de Responsabilidad Social en las empresas. La constitución de este ente obedece al objetivo de reunir en un mismo órgano a los representantes de los distintos grupos de interés vinculados con la Responsabilidad Social. El Ministerio de Empleo y Seguridad Social y la Dirección General del Trabajo Autónomo, de la Economía Social y de la Responsabilidad Social de las 
empresas, ostentan la Presidencia y Secretaría de CERSE respectivamente. Y esta última realiza además labores de preparación, apoyo técnico y gestión del Consejo.

El CERSE se crea en España para el desarrollo de la difusión de la información sobre el uso de criterios sociales, medioambientales y de buen gobierno, está compuesto por 56 representantes entre los que se cuentan los principales grupos de interés relacionados con la responsabilidad Corporativa: las Administraciones Públicas, las Empresas, las Organizaciones Sindicales y otras organizaciones de la Sociedad Civil, con 14 miembros cada uno.

En los últimos años la actividad normativa y regulatoria se ha visto reforzada a nivel europeo y mundial, apareciendo varios hitos fundamentales como son la Directiva Europea de Información No-Financiera (2013), la publicación del nuevo Código de Buen Gobierno de las Sociedades Cotizadas de la Comisión Nacional del Mercado de Valores (CNMV) (2015), la presentación de los Objetivos de Desarrollo Sostenible de la ONU (UNDP, 2015) o el Acuerdo contra el Cambio Climático derivado de la cumbre COP21 de Paris (2015).

Un aspecto importante que toma fuerza es la mejora del gobierno corporativo y su transparencia ya que son los cimientos sobre los que deben apoyarse las empresas para desarrollar correctamente la RSC, por lo que el compromiso y la convicción de los máximos órganos de gobierno de las empresas en esa tarea es fundamental y condición sine qua non para su desarrollo (OLCESE, 2013). Este aspecto queda reforzado en el "Estudio Multisectorial sobre el estado de la Responsabilidad Corporativa de la gran empresa en España" (2016), correspondiente al año 2015. Este estudio es un cuestionario a un total de 125 empresas, de las cuales 28 cotizan en el IBEX-35, agrupadas en 10 sectores de actividad. De sus resultados se extrae el crecimiento positivo experimentado en referencia a la información que se presenta a la alta dirección en materia de Responsabilidad Corporativa (Figura 3).

Figura 3. Cuadro de mando integral y síntesis de indicadores de RSC 
¿Existe un Cuadro de Mando Integral o similar que se presenta periódicamente a la Alta Dirección y sintetiza los indicadores de responsabilidad corporativa?

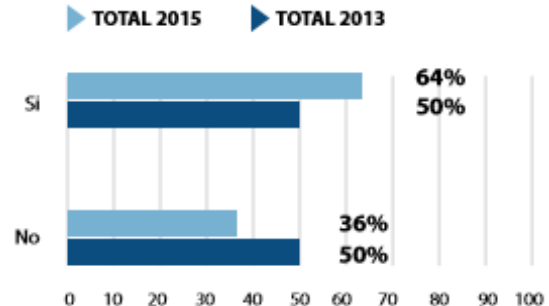

- El $64 \%$ de las empresas cuenta con un Cuadro de Mando Integral o similar que se presenta periódicamente a la Alta Dirección y sintetiza los indicadores de RC.

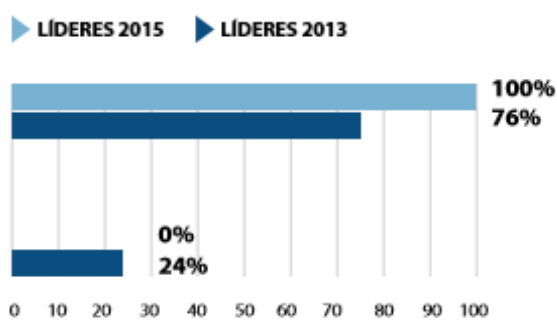

- La totalidad de las empresa líderes tienen un Cuadro de Mando Integral o similar que se presenta periódicamente a la Alta Dirección y sintetiza los indicadores de RC.

Fuente: Estudio multisectorial sobre el estado de la Responsabilidad Corporativa de la gran empresa en España" (2016)

En cuanto a los medios empleados para transmitir la comunicación de la responsabilidad Social, se observa la utilización de multicanales y unos porcentajes de utilización significativos que van aumentando año tras año, donde priman la utilización de las webs corporativas, otros medios en internet y la participación en foros y estudios con otras empresas u organizaciones (Figura 4).

Figura 4. Canales de comunicación de la RSC

¿Cómo se comunican las prácticas de responsabilidad corporativa? La empresa pone a disposicıón de sus grupos de Interés información sobre responsabilidad corporativa en alguno -o todos- de los sigulentes canales?

Pregunta multiopclón

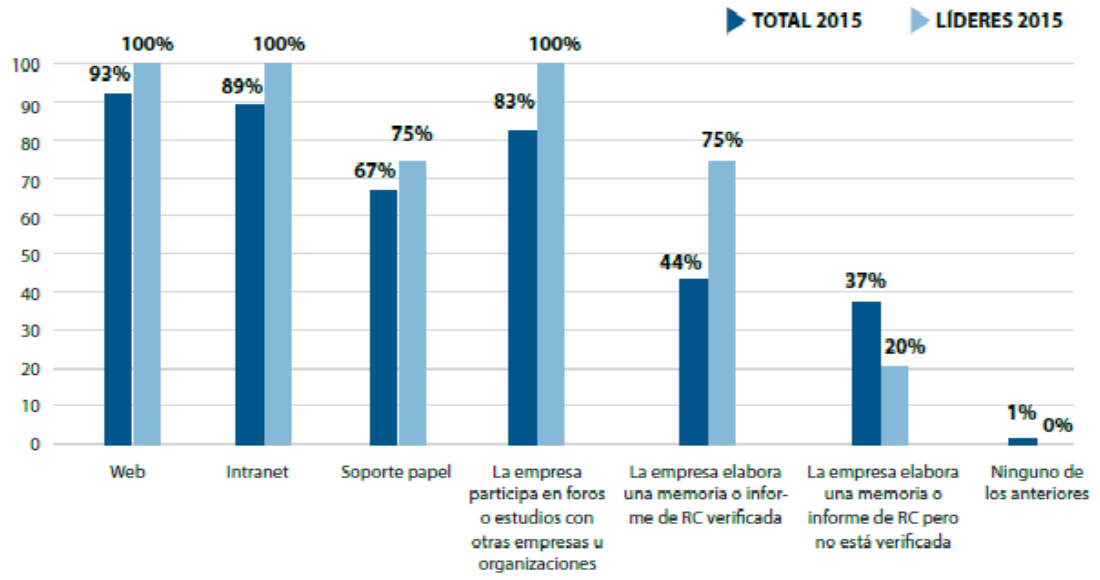

- Prácticamente todas las empresas comunican sus prácticas de responsabilidad corporativa a través de la Web y de la Intranet y además participan en foros o estudios con otras empresas u organizaciones. 
Fuente: Estudio multisectorial sobre el estado de la Responsabilidad Corporativa de la gran empresa en España" (2016)

Se muestra en el estudio analizado que las empresas optan en mayor medida por presentar en un único documento toda la información estratégica, económica, ambiental, social y de buen gobierno, en el espacio temporal considerado, siguiendo las directrices internacionales para informes integrados (Figura 5).

Figura 5. Informe integrado empresarial

¿La empresa presenta un Informe Integrado, que incluye las actuaciones de responsabilidad corporativa/ sostenibilidad?

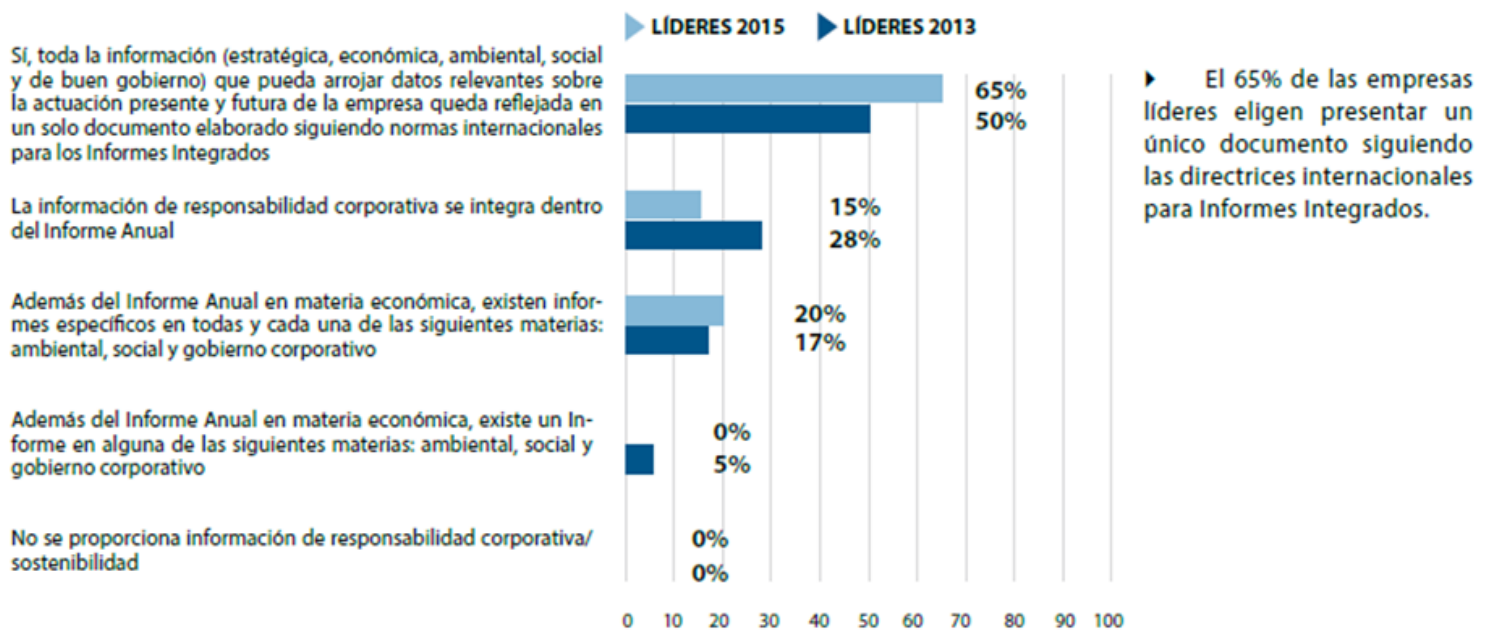

Fuente: Estudio multisectorial sobre el estado de la Responsabilidad Corporativa de la gran empresa en España" (2016)

El Club de Excelencia en Sostenibilidad (2016), en el "Estudio multisectorial sobre el estado de la Responsabilidad Corporativa de la gran empresa en España” (2016), evidencia que las empresas responsables tiene cada vez más éxito que el resto. Así, del estudio se extrae que la inclusión de la RSC en la estrategia de la empresa provoca que:

- Sus trabajadores sean más productivos y se sientan más identificados con ella, ya que perciben que la organización está alineada con la cultura predominante del respeto a las personas, al planeta y a la sociedad en general.

- Sus clientes les compren más, ya que esa cultura común es compartida, y defienden esa forma de hacer, con lo que se complementan y refuerzan su acción de responsabilidad. 
- La reputación mejore, ya que la sociedad aprecia esa labor de mejora social, tanto a nivel interno como externo, en su relación con los grupos de interés con los que interactúa.

- Reduzca sus costes internos y mejore su competitividad, ya que contratar o comprar a empresas responsables reduce riesgos y ese riesgo tiene un alto coste en la empresa.

\section{CONCLUSIONES}

La RSC se debe entender como una vía para lograr una mayor democracia económica, dando participación a los grupos de interés relacionados en las decisiones, y buscando un equilibrio entre la eficiencia y la equidad. Ayudando, mediante su implantación, al Estado en la gestión de los fallos del mercado y las externalidades, compensando la información y los mercados imperfectos y la limitada capacidad reguladora de la administración.

La gestión empresarial responsable ya no es aplicable simplemente al campo económico, ni pretende únicamente la satisfacción de las necesidades físicas de los individuos, sino que se convierte en una doctrina, unas normas y unos valores con una finalidad eminentemente social, donde asume su responsabilidad económica a través del compromiso con esos valores, creencias y normas, para la construcción de una relación estable y duradera con la sociedad y sus individuos, basada en la confianza y la estima mutua. Para lograr ese cambio se debe asumir un comportamiento empresarial verdaderamente responsable, donde se perciba claramente la necesidad de cambio, que la motivación para ese cambio sea fuerte, que los objetivos sean claros y precisos, que exista un liderazgo fuerte y agentes de cambio, que se establezcan planes de acción y mecanismos de control, tanto del proceso como de sus resultados, que permitan la mejora continua.

La asunción de la RSC implica la aplicación del buen gobierno corporativo, lo que convierte a la empresa en una figura responsable y ciudadana, interiorizando características básicas como disciplina, transparencia, independencia, rendición de cuentas, responsabilidad e imparcialidad. Por tanto, según estas premisas entendemos la RSC como un fin en sí misma y derivada de un deber asumido, creando una empresa más humana, consciente y comprometida con los problemas sociales y medioambientales de la comunidad y abordando preocupaciones sociales generales que contribuyan al desarrollo del entorno en el que actúa.

Para conseguir ese buen gobierno corporativo se pueden establecer unas pautas generales como son: 
- Su incorporación definitiva en la organización empresarial, en su misión, visión, cultura, filosofía, valores, políticas de gestión, etc.

- Asumir la eficiencia y la equidad como fines de la empresa: viabilidad, continuidad, estabilidad, competitividad, innovación y sensibilidad.

- Identificar e implicar a las partes interesadas, tanto internas como externas.

- Exigir la responsabilidad imprescindible de los administradores y alta dirección, a través de la creación de valor de modo sostenible, gestionando prudentemente, buscando el consenso y fomentando adhesiones entre grupos de interés, etc.

- Asimilar una organización, dirección y gestión participativa, que se traduzca en un diálogo continuo con los grupos de interés, adoptando reglas de juego consensuadas por las partes.

- Buscar la asignación y equilibrio de la distribución de la riqueza creada con una negociación justa, con ausencia de fuerza, fraude o manipulación, pero reconociendo las contribuciones, costes y riesgos asumidos por los partícipes.

- Interiorizar las externalidades y rendir cuentas con total transparencia, admitiendo la verificación externa independiente.

- Asumir un compromiso ético, de respeto de la diversidad y la igualdad de oportunidades, traducido en un código formal de RSC.

Sin embargo, todas las acciones implementadas por las empresas en materia de RSC podrían no conseguir el fin último deseado, a no ser que se realice una comunicación oportuna que lo ponga en conocimiento de los grupos de interés y la sociedad en general. La comunicación corporativa asume así un papel fundamental en esta misión de dar a conocer y difundir el trabajo realizado por las empresas, con el fin de alcanzar los objetivos que se plantean tanto económica como socialmente.

Mediante un desarrollo efectivo de la comunicación corporativa, las prácticas empresariales lograrán ser identificadas por la comunidad y sus consumidores. Con ello aumentará la consciencia del mercado que permitirá potenciar la diferencia de actuación, con respecto de aquellas que no sean socialmente responsables, y conseguir la ventaja competitiva perseguida. Comunicación entendida, no como el simple lanzamiento de mensajes, sino como agente cohesionador entre lo que se dice y lo que se hace y ser un canal activo y abierto con los grupos de interés. Para ello la comunicación estratégica debe establecer la estrategia para dicha conexión, mantener una actitud de escucha activa y permanente de los grupos de 
interés, asegurar el feedback que permita la comprensión efectiva de los mensajes emitidos e integrar e la estrategia comunicativa las respuestas.

\section{REFERÊNCIAS}

ANCOS, H. La responsabilidad social corporativa y sus actores: mitos y desafíos de la RSC. Madrid: Instituto Complutense de Estudios Internacionales, 2010.

ARGENTI, P. A. Corporate communication. New York: McGraw-Hill Higher Education, 2013.

ARVIDSSON, S. Communication of Corporate Social Responsibility: A Study of the Views of Management Teams in Large Companies. Journal of Business Ethics, v. 96, p. 339-354, 2010.

BRICEÑO, S.; MEJÍAS, I.; MORENO, F. La Comunicación Corporativa y la Responsabilidad Social Empresarial (RSE). Revista Daena (International Journal of Good Conscience, v. 5, n.1, p. 37-46. 2010. Disponível em:

<http://search.ebscohost.com/login.aspx?direct=true\&profile=ehost\&scope=site\&authtype=crawler\&jr $\mathrm{nl}=1870557 \mathrm{X} \& \mathrm{AN}=48757526 \& \mathrm{~h}=\mathrm{FYYBWv} 45 \mathrm{DAbieBUV} 5 \mathrm{NM} 4 \mathrm{mgK} 6$ oscLvMGRLvuNM2AJ0s40x 5URV8wzntCxJ8KIAC5FXFBC47fCa\%2BHiHplyQOWh6Q\%3D\%3D\&crl=c >. Acesso em: 21 set.

BRUSELAS. DIRECTIVA 2014/95/UE DEL PARLAMENTO EUROPEO Y DEL CONSEJO, de 22 de octubre de 2014, por la que se modifica la Directiva 2013/34/UE en lo que respecta a la divulgación de información no financiera e información sobre diversidad por parte de determinadas grandes empresas y determinados grupos Texto pertinente a efectos del EEE. Disponível em: <http://eurlex.europa.eu/legal-content/ES/TXT/?uri=CELEX\%3A32014L0095>. Acesso em: 15 set.

CARRILLO, S. Comunicación: clave para la gestión de la responsabilidad social. Conexión, v. 2, p. 132-149, 2015.

CLUB DE EXCELENCIA EN SOSTENIBILLIDAD. Estudio Multisectorial sobre el Estado de la Responsabilidad Corporativa de la Gran Empresa en España 2015. 2016. Disponível em: <http://www.clubsostenibilidad.org/main.asp?id_pagina=26>. Acesso em: 15 set.

COMISIÓN NACIONAL DEL MERCADO DE VALORES. Código de buen gobierno de las sociedades cotizadas. 2015. Disponível em:

https://www.cnmv.es/docportal/publicaciones/codigogov/codigo_buen_gobierno.pdf. Acesso em: 15 set.

ESPAÑA. LEY 2/2011, DE 4 DE MARZO, DE ECONOMÍA SOSTENIBLE. Boletín Oficial del Estado, n. 55, de 5 de marzo de 2011, p. 25033-25235.

ESPAÑA. REAL DECRETO 1469/2008, DE 5 DE SEPTIEMBRE, DE CREACIÓN DEL CERSE. Boletín Oficial del Estado, n. 229, de 22 de septiembre de 2008, p. 38470-38471.

GARRIDO, F. Comunicación Estratégica. Barcelona: Editorial Gestión 2000, 2004.

KOTLER, P.; HESSEKIEL, D.; LEE, N. Lo bueno funciona (Vol. 1). Madrid: LID Editorial, 2012.

MAZO, J. Estructuras de la Comunicación por Objetivos. Barcelona: Editorial Ariel, 2001.

MOURIZ, J. Comunicación Corporativa. 2007. Disponível em: <http://mouriz.wordpress.com/2007/06/06/rsc/>. Acesso em: 15 set. 
OLCESE, A. (Dir). Informe sobre la Responsabilidad Social de la Empresa en España. Una Propuesta para Europa. Bruselas: Comisión Europea. 2013. Disponível em:

<http://www.conr.es/sites/default/files/archivos/Informe\%20sobre\%20la\%20responsabilidad\%20socia $1 \% 20 \mathrm{de} \% 201 \mathrm{a} \% 20 \mathrm{empresa} \% 20 \mathrm{en} \% 20 \mathrm{Espa} \% \mathrm{C} 3 \% \mathrm{~B} 1 \mathrm{a} . \mathrm{pdf}>$. Acesso em: 20 set.

PIZZOLANTE, Í. El Poder de la Comunicación Estratégica. Caracas: Editorial CEC, 2006.

UNDP. Objetivos de desarrollo sostenible. 2015. Disponível em:

<http://www.undp.org/content/undp/es/home/sustainable-development-goals.html>. Acesso em: 15 set.

UNIÓN EUROPEA (UE). Libro Verde Fomentar un marco europeo para la responsabilidad social de las empresas. Bruselas: Comisión Europea. 2001. Disponível em: <http://eurlex.europa.eu/legalcontent/ES/TXT/PDF/?uri=CELEX:52001DC0366\&qid=1433318086120\&from=EN>. Acesso em: 15 set.

UNIÓN EUROPEA (UE). Guía para una comunicación eficaz. Bruselas: Comisión Europea, Dirección General de Empresa. 2005. Disponível em: <http://rscat.gencat.cat/web/.content/05__actualitat/06-documents/noticias/arxius/guia_para_una_comunicacion_eficaz.pdf $>$. Acesso em: 15 set.

VÁZQUEZ, D. G.; POLO, F. C. Diseño de un sistema de comunicación eficaz para la responsabilidad social en pymes. Información Comercial Española, ICE: Revista de economía, v. 872, p. 161-178, 2013.

VICENTE, A.; BALDERAS, A.; RUIZ, M.; TAMAYO, U. Responsabilidad social en la empresa y competitividad. Xertatu. 2007. Disponível em:

<http://www.bizkaia.eus/home2/archivos/DPTO8/Temas/xertatu/archivos/revista1.pdf?hash=ee2d016 e89ca835d2db2ba224c67a406\&idioma=CA >. Acesso em: 21 set.

VIDAL, N.G.; BULL, G.Q.; KOZAK, R.A. Diffusion of Corporate Responsibility Practices to Companies: The Experience of the Forest Sector. Journal of Business Ethics, v. 94, p. 553-567, 2010.

Original recebido em: 22 de setembro de 2017

Aceito para publicação em: 30 de novembro de 2017

María Concepción Parra-Meroño

Departamento de Ciencias Sociales, Jurídicas y de la Empresa

Phd. Marketing y Organuzación de Empresas

Directora del Master Universitário en Marketing y Comunicación

José Gabriel Mira-Agulló

Departamento de Ciencias Sociales, Jurídicas y de la Empresa

Licenciado em Administración y Direción de Empresas

Esta obra está licenciada sob uma Licença Creative Commons. 


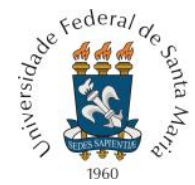

PROGRAMA DE PÓS-GRADUAÇÃO EM COMUNICAÇÃO DA UNIVERSIDADE FEDERAL DE SANTA MARIA 